\section{Trapping human genes}

\section{By Amy Donner, Senior Editor}

The Research Center for Molecular Medicine of the Austrian Academy of Sciences and Haplogen GmbH have generated a library of haploid knockout human cells that, for the first time, allows for the systematic interrogation of gene function in vitro. ${ }^{1}$ The partners are completing the library, although the collection already is available to the scientific community and is being used for Haplogen's internal drug discovery efforts.

In model organisms, gene inactivation has been a powerful approach to elucidate protein function. In particular, libraries of haploid yeast cells-with one gene inactivated per cell-have been invaluable to helping understand basic cellular processes, gene-gene interactions and gene-drug interactions.
"Having knockout lines for all human genes available as reagents that are delivered within days will fundamentally change the way we perform and plan experiments."
- Georg Casari, Haplogen GmbH observed phenotype. systematic analysis of gene function across the entire genome.

Brummelkamp performed that research when he was at the Whitehead Institute for Biomedical Research.

To generate an actual library that would allow researchers to investigate specific genes on demand, the team engineered the gene-trap construct to contain barcodes of unique DNA sequences to facilitate identification and isolation of individual knockout clones. Following random integration of the construct into thousands of cells, analysis of the resulting clones indicated that gene trapping resulted in nearcomplete gene inactivation.

In the majority of cells in the library, gene inactivation was reversible. According to Nijman, reversible expression is helpful because it allows a scientist "to rule out passenger mutations as the cause of an interesting phenotype that emerges from a screen" and thus is a simple way to establish a functional connection between the trapped gene and the

With the right gene-trap insertion, essential genes could also be targeted without killing the cells-a feature that improves the utility of the platform in research.

To validate the utility of the collection, the scientists showed that cells with mutations in genes downstream of multiple cytokines were no longer responsive to the proteins. The cytokine genes included tumor necrosis factor- $\alpha($ TNF- $\alpha)$ and interferon- $\gamma$ (IFNG; IFN$\gamma)$, the ligand TNF-related apoptosis-inducing

"For scientists working with human cells, however, there are no single knockout libraries available. We use RNAi or genome engineering strategies, but there is no equivalent to yeast libraries for mammalian cells," noted Sebastian Nijman, a principal investigator at the Research Center for Molecular Medicine (CeMM) and cofounder of Haplogen.

A group led by Nijman, Thijn Brummelkamp and Giulio SupertiFurga set out to generate the first library of human cells comparable to what is available to the yeast community. Brummelkamp is group leader at The Netherlands Cancer Institute, adjunct principal investigator at CeMM and cofounder and head of research at Haplogen. Superti-Furga is $\mathrm{CEO}$, scientific director and a principal investigator at CeMM and cofounder of Haplogen.

The biggest hurdle was the difference in genome copy number between yeast and humans. Unlike mammalian cells, haploid yeast occur naturally, which makes it easier to generate gene-specific knockouts. The human genome is diploid-cells contain two copies of every chromosome-which complicates the generation of gene-specific knockouts in cells.

Prior studies by Brummelkamp had provided proof of concept for using a gene-trap retrovirus to inactivate single human genes in a natural, stable, near-haploid human cell line called KBM7. The cell line was subcloned from a patient with $B C R-A B L^{+}$chronic myelogenous leukemia (CML). ${ }^{2}$

Although the approach worked, it was both labor intensive and time consuming. Moreover, it did not allow for the creation of a library for the ligand (TRAIL) and the growth factor transforming growth factor- $\beta$ (TGFB; TGF $\beta$ ).

Results were published in Nature Methods.

\section{Going for full coverage}

The library reported in the paper so far covers about a third of all the library should double in size within 12 months. New clones will be added to the collection as they become available.

According to Nijman, "The main objective is to generate a collection to cover the entire genome."

Haplogen CEO and cofounder Georg Casari added that "our goal is to provide a library that has a knockout clone for each human gene that can be removed. Having knockout lines for all human genes available as reagents that are delivered within days will fundamentally change the way we perform and plan experiments. Many experiments will now be limited only by having the right question or idea rather than by overcoming technical obstacles associated with genetic experiments in human cells."

Nijman's lab is now using the library to evaluate the mechanism of action for drugs and to elucidate gene-drug interactions.

Haplogen will use the library internally for drug discovery and development. "We will be able to perform novel screens for drug targets, mostly in the area of infectious diseases. Those programs can fuel our drug development pipeline, which already contains several targets identified using haploid genetics," noted Casari. protein-coding genes. Work to complete the collection is ongoing, and 


\section{ANALYSIS}

The Human Gene Trap Mutant Collection was generated as a publicprivate partnership between CeMM and Haplogen and is available to the scientific community at http://clones.haplogen.org/.

"The collection is available pretty much without limits for academic research via a material transfer agreement," said Nijman. The library is also available to companies through a licensing agreement or partnership with Haplogen.

Nijman and Casari expect the completed library will soon be followed by related libraries from their team and others. New genome editing methodologies, including clustered, regularly interspaced short palindromic repeats (CRISPR) and transcription activator-like effector nucleases (TALENs), ${ }^{3}$ could also be used to generate gene-specific knockouts, and libraries generated with these alternative technologies will likely provide access to complementary capabilities. But Nijman pointed out that useful features of the current library such as reversibility and DNA barcoding might be difficult to recreate with these other technologies.

Haplogen is seeking licensing and partnership opportunities and already has undisclosed partnerships in a variety of areas, including cancer and metabolic disease.

The library is not patented. The gene-trapping technology is patented by the Whitehead Institute.

Donner, A. SciBX 6(37); doi:10.1038/scibx.2013.1018

Published online Sept. 26, 2013

\section{REFERENCES}

1. Bürckstümmer, T. et al. Nat. Methods; published online Aug. 25, 2013; doi:10.1038/nmeth.2609

Contact: Sebastian M.B. Nijman, Research Center for Molecular Medicine of the Austrian Academy of Sciences, Vienna, Austria e-mail: snijman@cemm.oeaw.ac.at

2. Carette, J.E. et al. Science 326, 1231-1235 (2009)

3. Cain, C. SciBX 6(4); doi:10.1038/scibx.2013.77

COMPANIES AND INSTITUTIONS MENTIONED

Haplogen GmbH, Vienna, Austria

The Netherlands Cancer Institute, Amsterdam, the Netherlands Research Center for Molecular Medicine of the Austrian Academy of Sciences, Vienna, Austria Whitehead Institute for Biomedical Research, Cambridge, Mass. 